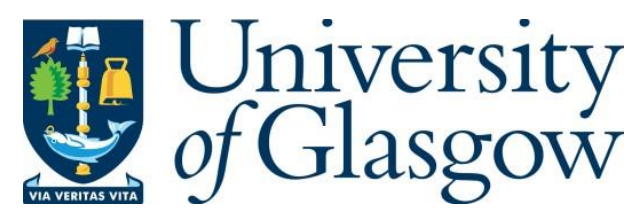

Wilson, L. S.J., Barker, J. R. and Martinez, A. E. (2019) DFT/NEGF study of discrete dopants in Si/III-V 3D FET. Journal of Physics: Condensed Matter, 31(14), 144003.

There may be differences between this version and the published version. You are advised to consult the publisher's version if you wish to cite from it.

http://eprints.gla.ac.uk/179083/

Deposited on: 5 March 2019

Enlighten - Research publications by members of the University of Glasgow

http://eprints.gla.ac.uk 


\title{
DFT/NEGF study of discrete dopants in Si/III-V 3D FET
}

\author{
L S J Wilson ${ }^{1}$, J R Barker ${ }^{2}$, A E Martinez ${ }^{1}$ \\ ${ }^{1}$ College of Engineering, Swansea University Bay Campus, Fabian Way, Swansea \\ SA1 8EN, UK \\ ${ }^{2}$ Rankine Building, School of Engineering, College of Science and \\ Engineering,University of Glasgow, Glasgow G12 8LT, UK
}

E-mail: l.s.j.wilson@swansea.ac.uk

\begin{abstract}
In this work, electron densities around dopants in $\mathrm{Si}$ and GaAs have been calculated using DFT calculations. These extracted densities have been used to describe dopants in an in-house Non-Equilibrium Green functions (NEGF) device simulator. The transfer characteristics of nanowire gate all around field effect transistor have been calculated using density functional theory (DFT) electron densities. These transport calculations were compared with those using a point charge model of the dopant. The dopants are located in the middle of the channel of the device. Specifically, DFT calculations of a 512 atom $\mathrm{Si}$ supercell with a single impurity atom have been carried out, both phosphorous and boron atoms have been used as donor and acceptor impurities respectively. The calculations were repeated on a gallium arsenide supercell, where the silicon atom substituted gallium and arsenide to act as donor and acceptor respectively. We found that for donors and acceptors, the DFT charge distribution extend similarly in both materials. In addition, the relaxed structure produces a $50 \%$ larger spread of electronic charge as compared with unrelaxed Si and GaAs. The extracted current voltage characteristics of the devices are altered significantly using the charge density obtained by DFT. At $0.7 \mathrm{~V}$ the current in $\mathrm{Si}$ is $20 \%$ larger using the DFT charge density compared to the point charge model for donors. Whereas the current using the point charge model in GaAs is 2.5 times larger than the distributed charge. Devices exhibit substantial tunnelling currents for donors and acceptors irrespectively of the model of the dopant considered. In GaAs, this was $76 \%$ using a point charge and $78 \%$ using the distributed charge when using a donor; $61 \%$ and $68 \%$ in Si respectively. The tunnelling current using acceptors for $\mathrm{Si}$ was $100 \%$ and 99\% using GaAs for both models.
\end{abstract}

Keywords: Density Functional Theory, Non-equilibrium Green Functions, Discrete Dopants 


\section{Introduction}

The continuous downscaling of metal-oxide-semiconductor field effect transistors (MOSFET) has been limited by short channel effects and poor electrostatic control of the gate. As a result, silicon ( $\mathrm{Si}$ ) nanowire field effect transistors (NWFETs) have been in production for several years and exhibit superior performance for low power devices and fast switching $[1,2]$. As the size of the active regions of devices continues to be scaled down to sub-micrometre dimensions, the number and location of a few impurities dramatically affect device performance between identically manufactured devices. Nanowires with various channel lengths and cross sections have been fabricated $[3,4,5]$. Tunnelling and confinement combined by strong local electrostatic potential invalidate the use of semi-classical models such as the drift-diffusion and Monte Carlo methods, however are still are applicable due to MOSFETs charge integrity blurring the quantum effects when tunnelling and confinement are negligible, but still require continuous calibration at specific dimensions to reliably model the transfer characteristics. The lack of validity of these methodologies at sub-10 nanometre dimensions is a direct consequence of the wave nature of the electron. The severity of quantum effects, such as tunnelling and confinement, only allows for purely quantum mechanical simulations to provide a reliable measure of electron transport. Substantial work on discrete dopants has been carried out using a discrete point charge dopant in the non-equilibrium Green functions (NEGF) formalism [6, 7, 8]. The electron density produced by the point charge model is mostly spherically symmetric or slightly deformed by the effective mass tensor. However, the wavefunction of the electron/hole occupying the dopant atom exhibits a tetrahedral symmetry and a shape dictated by the atomistic distribution of the surrounding atoms. A number of theoretical works have considered impurities in Si nanowires using Density Functional Theory (DFT) with an emphasis on different radial dopant positions. $[9,10,11]$, the scattering properties of a single phosphor donor in Si has previously been investigated. In recent years progress has been made in III-V semiconductors, particularly for photonics applications [12]. GaAs has a significantly lower intrinsic carrier concentration than Si, however it has garnered attention due to its larger mobility $\left(\mu_{n}\right)$ of around $8500 \mathrm{~cm}^{2} / \mathrm{V}$.s resulting in faster devices than what Si can achieve. With recent improvements in the quality of group III-V materials, fabrication of devices with these materials is now a real possibility. Transistors constructed from this material are researched intensively $[13,14]$. However, a key issue limiting the development of nanotransistors is device variability, which significantly alters device behaviour and limits integration with current CMOS technology [15]. Dopants in Si have also shown potential for qubits in solid-state computers, they can be scaled to large arrays controlled by a gate electrode $[16,17,18]$.

In this work, we investigate the charge distribution of impurity atoms in Si and GaAs along with the transfer characteristics of gate-all-around (GAA) NWFETs with Si and GaAs cores, comparing two impurity models (point charge and DFT). Specifically, Kohn-Sham densities were extracted from DFT calculations and input into an in-house 
device simulator to force the electron distribution to adopt the DFT distribution; This electron distribution was produced by solving the Poisson equation in the simulator. This paper is divided into the following sections: Section 2 gives a description of the DFT methodology used to calculate the electronic structure and extract the Kohn-Sham electron density to be used in a separate device simulator. A description of the NEGFPoisson computational models used to treat electron transport is presented. In section 3, we present our electronic structure results of 512 atom Si and GaAs supercells with dopant impurities and secondly our quantum transport calculations where the current voltage characteristics for different type of dopant models are presented. Our conclusions are drawn in section 4.

\section{Simulation Methodology}

\subsection{Density Functional Theory}

We have performed the calculation using ab initio electronic structure calculations with the SIESTA code (Spanish Initiative for Electronic Simulations with Thousands of Atoms) [19]. The code is based on the localized linear collection of atomic orbitals (LCAO) basis set to describe valence electrons and norm-conserving pseudopotentials for the atomic core. In this work, one particular generalized gradient approximation (GGA) has been used for the form of the exchange-correlation functional, PerdewBurke-Ernzerhof [20] which appears in the Kohn-Sham equations. GGA has been used previously with phosphorous doped Si systems to produce results that agree with experiments [21]. GGA has been used in all cases along with a double- $\zeta$ polarized basis set and atomic orbitals were described by norm-conserving Troulier-Martins pseudopotentials [22]. We relaxed the geometry of the doped materials until the forces are lower than $0.04 \mathrm{eV} / \AA$ using conjugate gradient optimization with Pulay algorithm for iteration mixing and were found to be converged with a mesh-cutoff of 100 Rydbergs. Many theoretical calculations have used standard density functional throry (DFT) calculations and it is well known that computing the band gap using Kohn-Sham valence band and conduction band eigenvalues systematically underestimates the band gap for most semiconductors [23]. The use of the GW approximation (GWA) has been used to successfully provide a more accurate band gap [24]. An example of the performance of DFT for the band gap of several semiconductors can be found in [25]. We are well aware that more advanced approaches could provide corrections, but it is beyond the scope of this paper.

\subsection{Non-equilibrium Green Functions}

As mentioned earlier, the steady state NEGF formalism was used to calculate the current voltage characteristics. We used an in-house device simulator, which solves equations 1 and 2 to find the retarded $\left(\mathrm{G}^{R}\right)$ and lesser Green $\left(\mathrm{G}^{<}\right)$functions. 


$$
\begin{aligned}
& \left(H-\Sigma^{R}\right) G^{R}=E G^{R}, \\
& G^{<}=G^{R} \Sigma^{<} G^{A} .
\end{aligned}
$$

Where $H$ is the Hamiltonian comprising the kinetic and potential energy of an electron and $\Sigma^{R}$ is the retarded self-energy. $E$ stands for the electron energy. These equations are usually called NEGF equations in the electronic simulation community. Equation (1) describes the causal dynamics of the electronic states via the retarded single electron Green function $G^{R}$. De-phasing dissipative processes lead to a non-Hermitian retarded self-energy $\Sigma^{R}$ that describes the level shifts (real part) and lifetime/broadening (imaginary part) of the electronic states. In addition, the self-energy is constructed to formally include a non-Hermitian component that accounts for the injection or extraction of electrons at the boundaries of the simulation domain. It is noted that $G^{R}$ yields the electronic spectral density of states. The second equation, for the lesser Green function $G^{<}$, describes the statistical dynamics (occupancies) of the electronic states and is akin to a quantum generalisation of the Boltzmann equation. $G^{<}$is related to a generalised Wigner distribution. Similarly, $G^{>}$represents the dynamical occupancies of vacancies (holes) in the electronic states at energy $E$. In equation (2) the lesser self-energy $\Sigma^{<}$is usually called the scattering-in self-energy and it represents the rate at which electrons enter a state of energy $E$. The number of electrons entering an unoccupied state of energy $E$ per unit time is given by $G^{>} \Sigma^{<}$.

There are as many equations (1) and (2), as energies, considered in the problem. The energy mesh used in this work contains more than 2000 points. So the problem is computationally intensive. The input to equations (1) and (2) is the electrostatic potential and Fermi levels at the source and the drain of the transistor. At the source and drain, the fluctuation-dissipation theorem has been used to write the contact selfenergies, this the standard way that is used in the NEGF device simulation [26]. Not all the elements of the $G^{R}$ and $G^{<}$are needed, only the diagonal and first off-diagona lterms (the latter corresponding to discretization of the kinetic energy component of the Hamiltonian in the position representation). Discretization of the current equation and details of the recursive algorithm applied to NW transistors can be found in [26].

This allows the use of a recursive algorithm to calculate the required elements. This substantially increases the speed of computation and reduces the memory footprint. If scattering is considered, the corresponding scattering parameters and self-energies should be provided. From the lesser Green function, the density and current for an electron with energy $E$ are calculated by the following equations:

$$
\begin{aligned}
& n(E, x)=i G^{<}(E, x, x) \\
& J(E, x)=-\left.i \frac{e \hbar}{2 m}\left(\frac{\partial}{\partial x}-\frac{\partial}{\partial x^{\prime}}\right) G^{<}\left(E, x, x^{\prime}\right)\right|_{x=x^{\prime}}
\end{aligned}
$$

This expression only required the diagonal and first off diagonal element of $G^{<}$. When using equations (3) and (4) an additional integration over the energy is required 
to calculate the total electron density and current density at the point x. Equation (4) is the current in the x-direction. Our simulation used a mode space [27] to discretise the Hamiltonian. In this discretisation, the device region is split in cross-sections perpendicular to the transport directions (in our case is the axis of the nanowire). For each cross section along the wire, we calculated the eigenvalues (modes) and eigenfunctions (transversal wave functions). Now we expand the Hamiltonian in the modes of the cross-sections and in the positions along the wire axis (called $\mathrm{x}$ in this work). By picking few modes (under an energy cutoff) the size of the problem is substantially reduced as compared with the used of the full $\mathrm{x}, \mathrm{y}, \mathrm{z}$ or spatial basis. This mode space formulation produces similar results that the full 3D spatial discretization [28].

The kinetic energy in the $H$ is written in the effective mass approximation for general ellipsoidal band structures and the potential energy is given by the electrostatic potential. Equations (1) and (2) are solved in conjunction with Poissons equation. This last equation is necessary in order to calculate the electrostatic potential from the charge density and boundary conditions.

\section{Results}

\subsection{DFT Results}

In this section we focus on the effect of the charge distribution of a dopant impurity in the performance of a GAA NWFETs. The impurity atoms charge distribution from DFT is compared with that of a point charge. The Si and GaAs NWFETs have a [100]

core, gate oxide is $0.8 \mathrm{~nm}$ thick. Both source and drain are doped at $10^{20} \mathrm{~cm}^{-3}$, the channel has a single dopant impurity and is $6 \mathrm{~nm}$ long. The results are split into two sections, firstly, calculations of the electronic densities for DFT results and lastly the current voltage characteristics of devices with different dopant cases and models are calculated using a NEGF based device simulator.

The results of the electronic structure calculations performed on a single donor in Si and GaAs each with a 512 atom supercell are shown in figure 1a and figure 1b. Only one size of supercell is shown as we are interested in the electron distribution around the impurity, changing the supercell size will affect the energy, but the wavefunction less so. As we are comparing two models, the dopant was placed in the centre of the channel. This location maximizes the impact on transfer characteristics [29]. The isovalues were chosen to display the tetragonal symmetry of each lattice.

We have calculated the Kohn-Sham orbitals of the donor impurity in both cases. figure 2 shows the distribution of the Kohn-Sham orbitals with and without relaxation, where there is a more noticeable confinement of the distribution and both structures exhibit a similar distribution.

The radial probability, figure 2 , has been computed by evaluating the wavefunction around the site containing the donor and acceptor impurity, plotted against radial distance from donor site. The radial probability is large below $0.2 \mathrm{~nm}$. The radial 


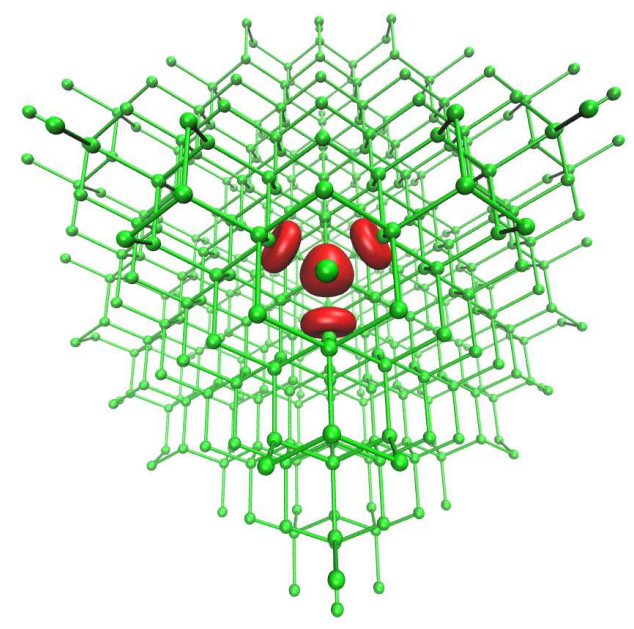

(a)

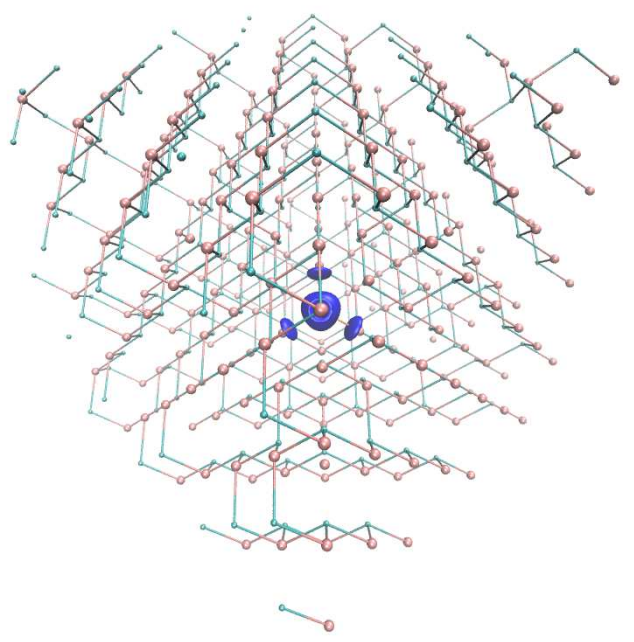

(b)

Figure 1: Group V donor in silicon where the electron density of the HOMO in the 512 atom supercell is centred on the phosphorous donor, (b) Silicon donor in GaAs where the electron density of the HOMO in the 512 atom supercell is centred on the Si atom.



(a)

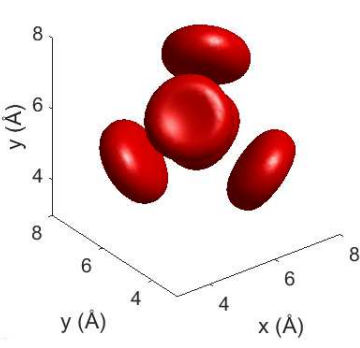

(b)

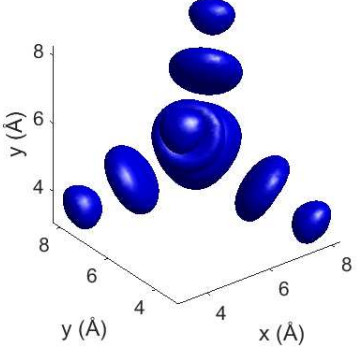

(c)

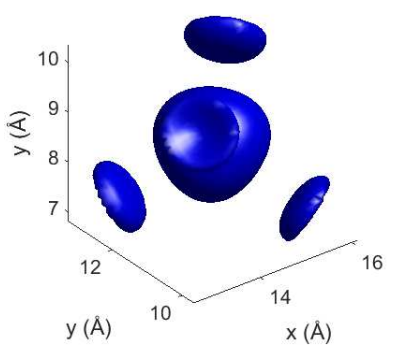

(d)

Figure 2: The probability density $\left(|\Psi|^{2}\right)$ for the donor electron. The wavefunction has been calculated using a supercell of 512 atoms. (a) Si unrelaxed, (b) Si relaxed, (c) GaAs unrelaxed and (d) GaAs relaxed. Si isovalue $=0.062$, GaAs isovalue $=0.38$.

probability for silicon below $0.2 \mathrm{~nm}$ is $17.16 \%$ when unrelaxed and $15.99 \%$ when relaxed. Unrelaxed GaAs has a probability of $16.94 \%$ unrelaxed and $8.33 \%$ relaxed. Using DFT we have calculated the electron density around Si and GaAs using both acceptor and donor impurities, these results are used in the next section to describe the dopant impurity and compare with that of a point charge. The corresponding acceptor impurity radial probabilities are shown in figure 3(b) for Si and GaAs, only unrelaxed are shown. However, our transport simulations do not show differences between relaxed and unrelaxed. 


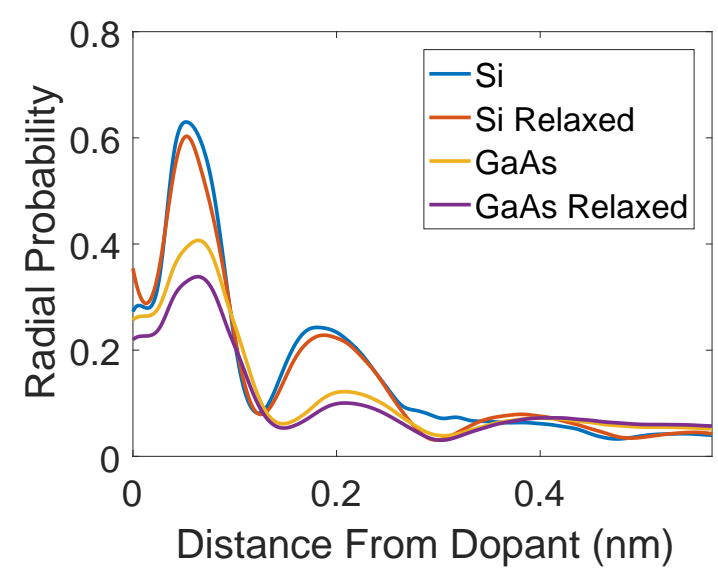

(a)

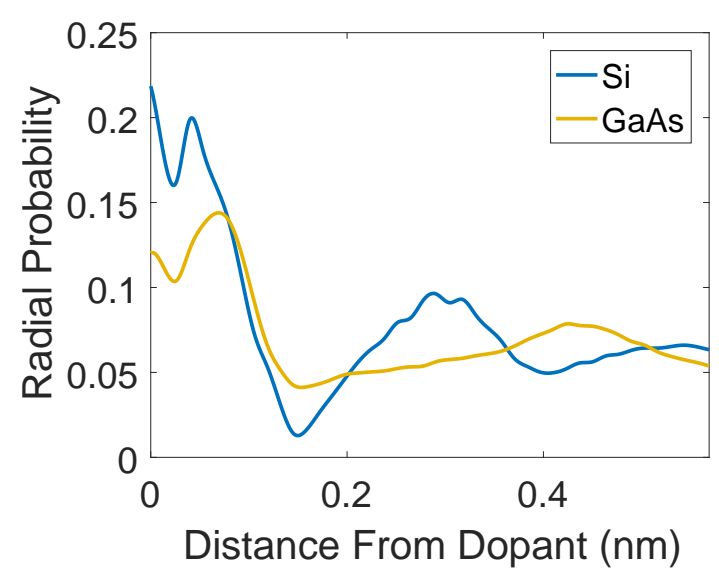

(b)

Figure 3: $\Psi^{2}$ as a function of the distance from the dopant, of the electrons ground state for Si and GaAs. The maximum of the probability has been normalized to one (a) Donor (b) Acceptor (unrelaxed).

\subsection{NEGF Results}

In this section, a comparison between the point charge and DFT distributed charge has been made for the NWFET. The current voltage characteristics of a nanowire transistor with a dopant in the middle of the channel have been studied. All simulations are performed at a $0.4 \mathrm{~V}$ drain bias. We considered four different cases: (i) Silicon NWFET with a donor, (ii) silicon nanowire NWFET with an acceptor, (iii) GaAS with a donor, (iv) GaAs NWFET with acceptor. We calculated the percentage change in drain current due to different dopant charge models.

The point charge model distributed the donor or acceptor charge by $0.2 \times 0.2 \times 0.2$ $\mathrm{nm}^{3}$. The DFT charge density is extracted from the square of the wavefunction obtained from the post-processing utility DENCHAR, bundled with SIESTA. The square of the wavefunction and it's associated volume were transferred to the mesh used in the NEGF simulator as a charge density; this was normalized to one electron charge in the NEGF discretization volume, therefore the total charge density adds to the charge of one electron. In all simulations we have used the finite volume discretization.

The $\mathrm{I}_{D^{-}} \mathrm{V}_{G}$ characteristics for the GaAs and Si transistors for the donor impurities are shown in figure 4 . The semi-log plot shows the behaviour on the NWFETs in the sub-threshold region. At low gate bias, $0.2 \mathrm{~V}$, the current of the GaAs device with point charge is 2.5 times larger as compared to the distributed charge. The precise value of the current at this bias are $6.89 \times 10^{-10} \mathrm{~A}$ and $2.679 \times 10^{-10} \mathrm{~A}$, for point and distributed charges respectively. This result is related with the fact that a point charge seems to be more efficient in lowering the source drain barrier and therefore producing large current. At high bias, $0.7 \mathrm{~V}$, the difference between models is negligible. However, in Si the large mismatch between the transversal wave functions dominates and the current is larger 


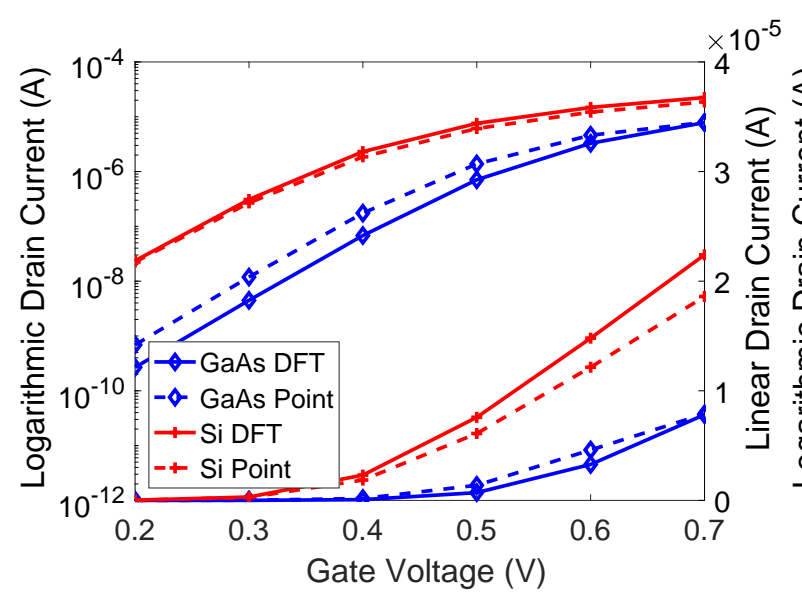

(a)



(b)

Figure 4: $\mathrm{I}_{D^{-}} \mathrm{V}_{G}$ characteristics of $\mathrm{Si}$ and GaAs GAA nanowires with point/distributed (DFT) charge density, $\mathrm{V}_{d}=0.4 \mathrm{~V}$, cross-section $=2.2 \times 2.2 \mathrm{~nm}^{2}$ (a) donor impurity (b) acceptor impurity

for the distributed charge case, even if the barrier is higher for the distributed charge model as compared to the point charge case. The distributed charge model produces a larger current of $5 \%$ and $20 \%$ for low and high gate bias respectively, compared to the point charge. If the overlap between transversal states is ignored then the current for the point charge case is large $\left(\mathrm{I}=1.22 \times 10^{-7} \mathrm{~A}\right)$ than the corresponding distributed charge case $\left(\mathrm{I}=3.20 \times 10^{-8} \mathrm{~A}\right)$ as expected. This implies that the height and width of the barrier is not the only factor influencing the current. For the GaAs cases, the height of the barriers seems to be the dominant effect. At high gate bias, the barrier is affected by electrostatic self-consistency, as a substantial amount of electrons are attracted to the channel. It should be noted that due to the short channel length a large amount of electrons cross the barrier by tunnelling.

For the case of an acceptor impurity at low gate bias, $0.2 \mathrm{~V}$, the current is lower for both cases using the point charge. In this case the use of point charge model substantially increases the barrier high at low gate bias producing a decrease in current respect to the DFT case. In GaAs, the current is $96 \%$ larger and in Silicon is $66 \%$ larger than the current using the point charge model. It is also important to realize that there is source to drain tunnelling which also affects the electron transfer through the dopant barrier. Figure 5, shows the first subband profiles for all the cases studied along the channel of the device at $0.2 \mathrm{~V}$ gate bias. Comparing the donor cases for Si and GaAs we see that Si shows a more asymmetric well for the same bias condition as there is a large threshold voltage for the GaAs devices (see figure 4). The barrier height is approximately $0.2 \mathrm{~V}$ and $0.3 \mathrm{~V}$ for $\mathrm{Si}$ and GaAs respectively. However, for both cases the barrier is large for the distributed donor as explained before. For the case of the acceptor in Si, figure 5c. the top of the two barriers differ by $10 \mathrm{meV}$ which is enough to produce a substantial 


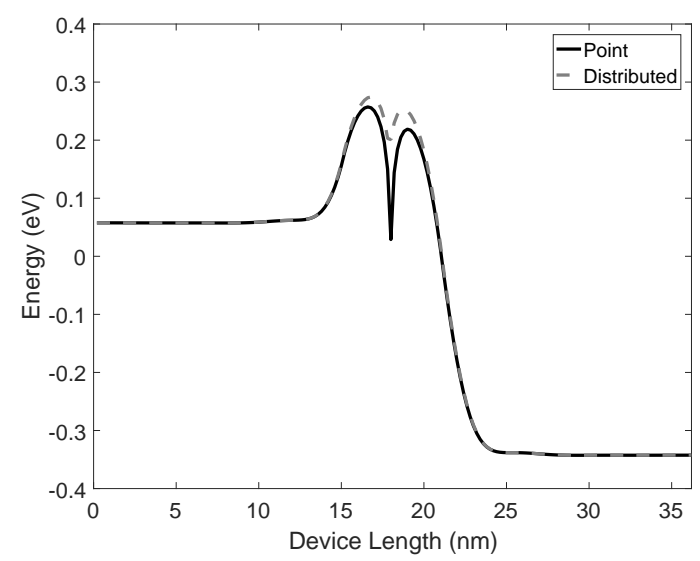

(a)

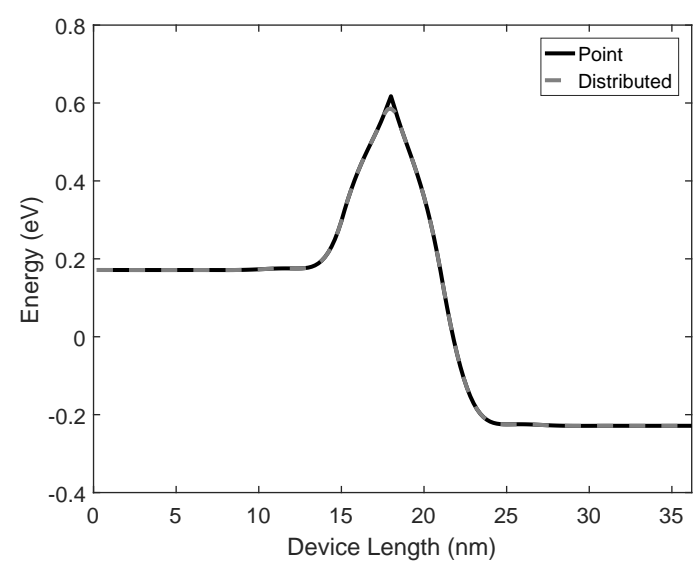

(c)

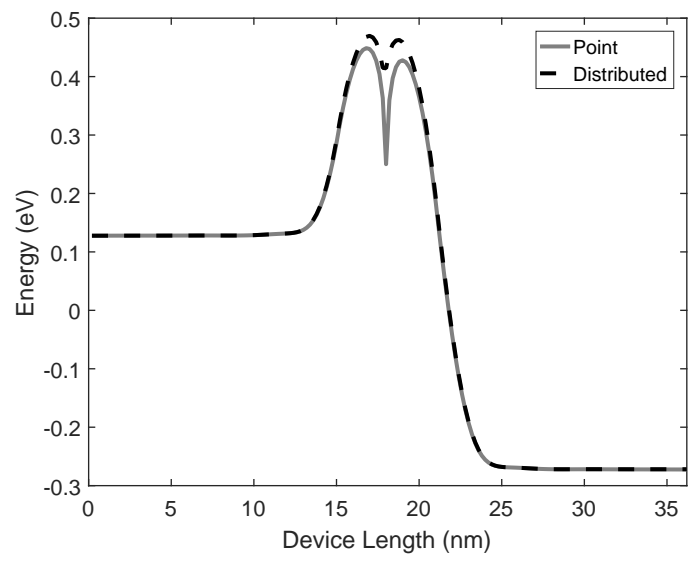

(b)

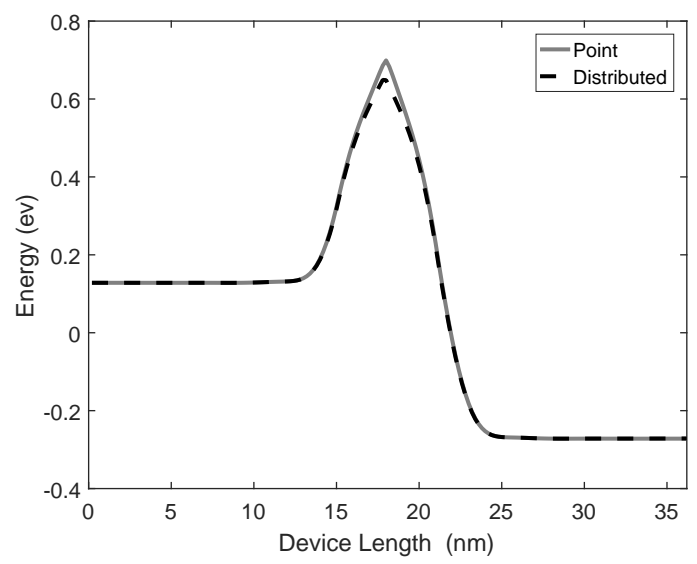

(d)

Figure 5: Comparison of the lowest energy subband between a point and distributed charge model, $\mathrm{V}_{g}=0.2 \mathrm{~V}$ (a) Si donor, (b) GaAs donor (c) Si acceptor (d) GaAs acceptor.

difference as the current goes exponential with the bias.

Figure 6 shows the corresponding current spectra along the Si device with the donor impurity. The tunneling current is calculated by integrating equation 4 up to the top of the sub-band energy. In both cases, punctual and distributed, there is a large tunnelling current. This tunnel current is $1.37 \times 10^{-} 8 \mathrm{~A}$ and $1.61 \times 10^{-} 8 \mathrm{~A}$ respectively, $61 \%$ and $68 \%$ of the total current respectively. The subbands corresponding to the three $\mathrm{X}$ valleys in silicon are shown. A shift larger than $0.1 \mathrm{eV}$ is observed between the first and second valleys, therefore the main contribution is given by the first valley as the thermal energy is roughly $26 \mathrm{meV}$.

Figure 7 a shows a 1D cut of the current spectrum for Si devices with the punctual and distributed donor cases. All current under the maximum value is tunnelling current, this confirm the large tunnelling in these devices. However, tunnelling is larger in the 


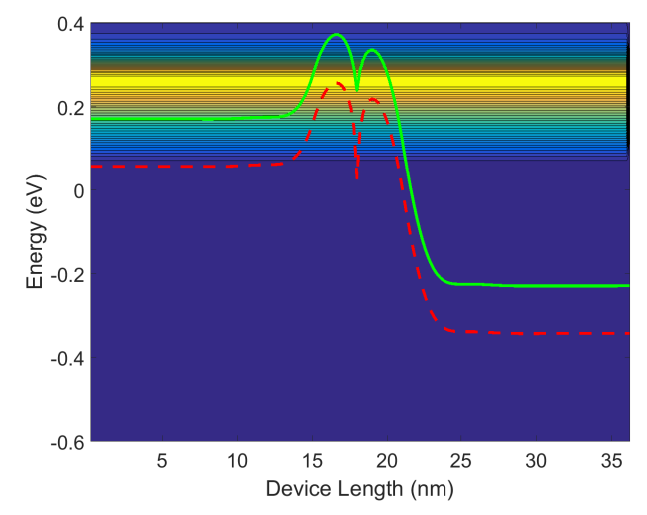

(a)

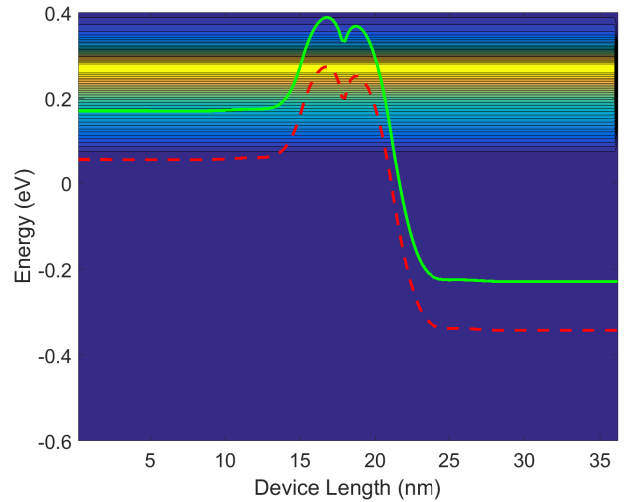

(b)

Figure 6: Energy-resolved current spectra for Si and sub-bands corresponding to three Valleys of Si, the lowest energy sub-band contains the first two degenerate valleys

(a) Point (b) Distributed. $\left(\mathrm{V}_{g}=0.2 \mathrm{~V}\right)$.

distributed device close to the top of the barrier due to better matching of the crosssectional wave functions. For the GaAs case shown in figure $7 \mathrm{~b}$ the current is larger for the point charge as the barrier is higher, figure $5 \mathrm{~b}$, and the mismatch plays a lesser role. Note the resonant peak in the figure.

On the other hand, for acceptor cases the current is larger than for the distributed case as the barrier height is smaller. In this cases as there are no resonant structures (see figure $5 \mathrm{c}$ and $5 \mathrm{~d}$ ), the current increases as the source-drain barrier decreases. The corresponding 1D cuts of the current spectrum are shown in figures $7 \mathrm{c}$ and $7 \mathrm{~d}$ for Si and GaAs respectively. In general, the barrier is larger for the point charge and therefore the current is reduced as compared with the DFT charge distribution. However, as mentioned before there is substantial tunnelling and reflection induced by the mismatch of the transversal wave functions, so it is very difficult to quantise each of these phenomena.

Finally, the density of states (DOS) along the channel for devices with a distributed donor impurity are shown in figure 8. In Figure 8a the low sub-band which appears corresponds to 4 equivalent valleys in $\mathrm{Si}$ which has longitudinal and transversal masses in the plane of the cross-section. The other two valleys have only transversal masses in the plane of the cross-section. As the transversal masses are smaller these two valleys appear higher in energy. However in the direction of transport, these valleys have a large mass (longitudinal) and therefore have closer DOS fringes. Similarly, the four low energy valleys have a small mass (transversal mass) in the transport direction which makes the fringe more separated. The resonant level at the middle of the channel is clearly visible. In GaAs, figure 8b,the gamma, $\mathrm{X}$ and $\mathrm{L}$ valley are all shifted respect to each other for details of the energy wavector dispersion see [30]. From low to high energy, the valleys are $L, X$ and $\Gamma$. 


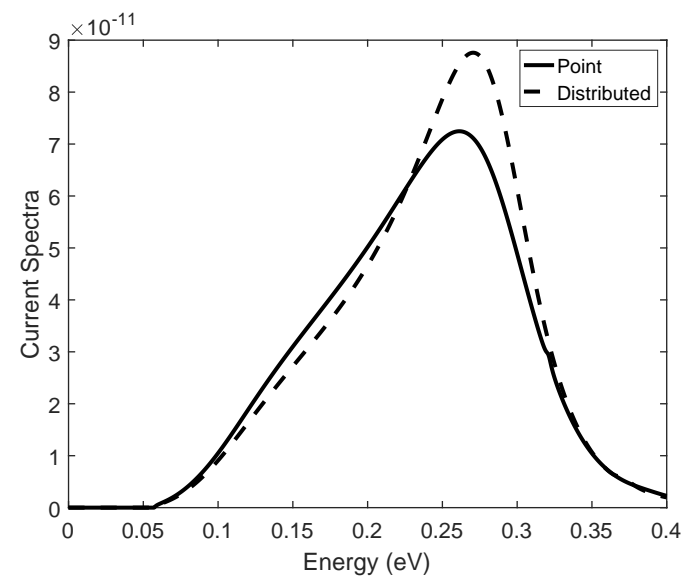

(a)

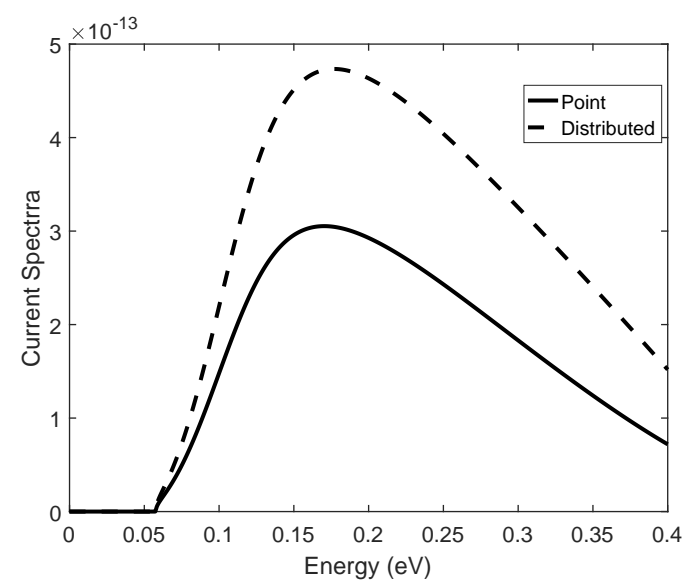

(c)

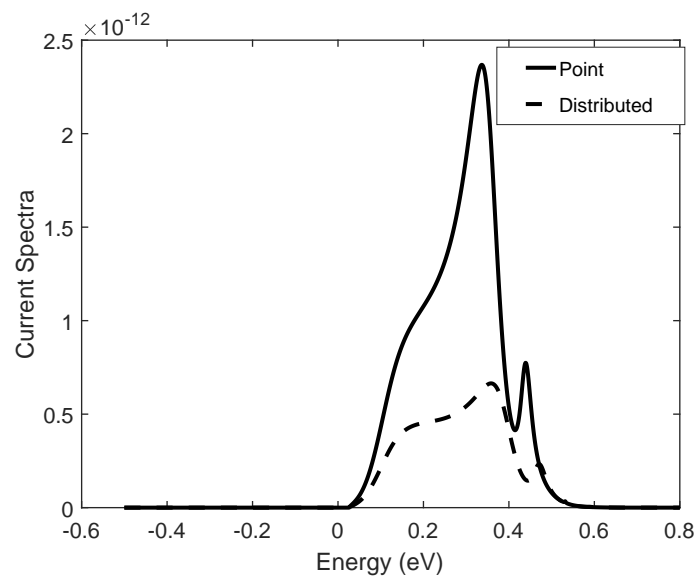

(b)

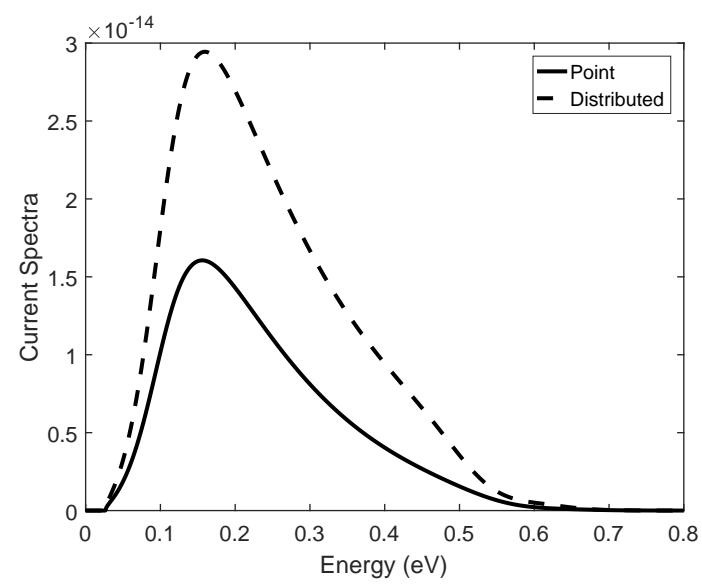

(d)

Figure 7: Comparison of the current spectra between a point and distributed charge model (a) Si donor, (b) GaAs donor (c) Si acceptor (d) GaAs acceptor. $\left(\mathrm{V}_{g}=0.2 \mathrm{~V}\right.$ ).

\section{Conclusion}

In this work, a combined DFT/NEGF method has been deployed to produce an accurate description of dopants in small nanotransistors with Si and GaAs cores. DFT calculations were carried out and charge distributions for donors and acceptors were extracted from the wave-functions. The calculation shows that the charge distribution in $\mathrm{Si}$ is less extended than for the GaAs device, however this difference is very small, the charge is $50 \%$ more distributed when relaxed for GaAs, but in Si this remains similar. The DFT extracted charge distributions were introduced in a middle of a channel of a GAA NW transistor in order to mimic the effect of the electron distribution around the impurities. Current-Voltage characteristics for a Si and GaAs devices with donor or acceptor were calculated using the DFT charge distribution and the commonly used punctual model. We found that in the case of acceptors, the DFT charge produced a larger current in both $\mathrm{Si}$ and GaAs; the off current is $66 \%$ and $96 \%$ larger and the on 


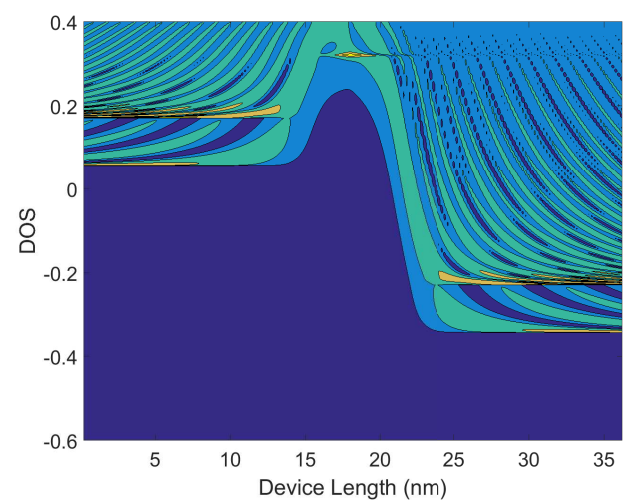

(a)

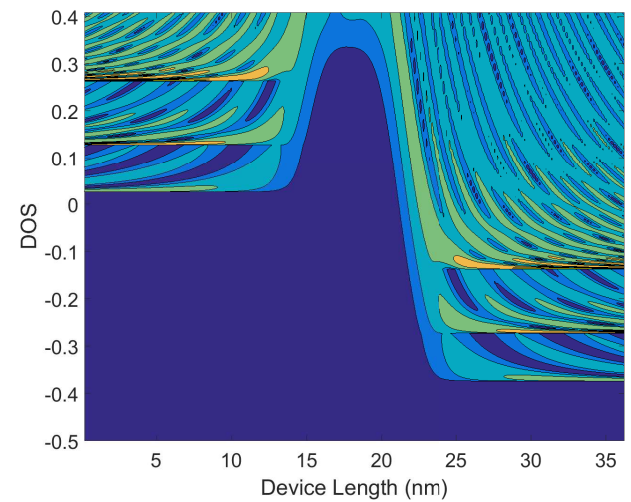

(b)

Figure 8: DOS of a device with a DFT donor impurity for (a) Si (b) GaAs. $\left(\mathrm{V}_{g}=0.2\right.$ $\mathrm{V})$.

current is $20 \%$ and $0.9 \%$ larger for $\mathrm{Si}$ and GaAs respectively. For the donor cases the situation is more complicated as the overlap between transversal states plays a strong role in the Si device. This mismatch of transversal states for the punctual case cause strong reflections lowering the transmission and therefore the current. For the case of GaAs donors, this effect is less and the height of the barrier dominates, producing large current for the punctual distribution. In general, we have demonstrated that for small nanotransistor an accurate description of the off current and on current will required a more sophisticated model for dopants beyond the simple point charge model.

\section{Acknowledgments}

Ricardo Rurali, Shirin Seifi

\section{References}

[1] Basudev Nag Chowdhury and Sanatan Chattopadhyay. Modeling of transport behavior of the ballistic silicon nanowire gate-all-around field-effect-transistors (si nwfets) with si/sio 2 interface roughness. In Computers and Devices for Communication (CODEC), 2012 5th International Conference on, pages 1-4. IEEE, 2012.

[2] M Aldegunde, A Martinez, and A Asenov. Non-equilibrium green function analysis of cross section and channel length dependence of phonon scattering and its impact on the performance of si nanowire field effect transistors. Journal of Applied Physics, 110(9):094518, 2011.

[3] Sung Dae Suk, Ming Li, Yun Young Yeoh, Kyoung Hwan Yeo, Keun Hwi Cho, In Kyung Ku, Hong Cho, WonJun Jang, Dong-Won Kim, Donggun Park, et al. Investigation of nanowire size dependency on tsnwfet. In Electron Devices Meeting, 200\%. IEDM 200\%. IEEE International, pages 891-894. IEEE, 2007.

[4] N Singh, FY Lim, WW Fang, SC Rustagi, LK Bera, A Agarwal, CH Tung, KM Hoe, SR Omampuliyur, D Tripathi, et al. Ultra-narrow silicon nanowire gate-all-around cmos devices: Impact of diameter, channel-orientation and low temperature on device performance. In Electron Devices Meeting, 2006. IEDM'06. International, pages 1-4. IEEE, 2006. 
[5] KH Cho, KH Yeo, YY Yeoh, SD Suk, M Li, JM Lee, M-S Kim, D-W Kim, D Park, BH Hong, et al. Experimental evidence of ballistic transport in cylindrical gate-all-around twin silicon nanowire metal-oxide-semiconductor field-effect transistors. Applied Physics Letters, 92(5):052102, 2008.

[6] R Valin, A Martinez, and JR Barker. Non-equilibrium green functions study of discrete dopants variability on an ultra-scaled finfet. Journal of Applied Physics, 117(16):164505, 2015.

[7] Antonio Martinez, Manuel Aldegunde, Natalia Seoane, Andrew R Brown, John R Barker, and Asen Asenov. Quantum-transport study on the impact of channel length and cross sections on variability induced by random discrete dopants in narrow gate-all-around silicon nanowire transistors. IEEE Transactions on Electron Devices, 58(8):2209-2217, 2011.

[8] Manuel Aldegunde, Antonio Martinez, and John R Barker. Study of discrete doping-induced variability in junctionless nanowire mosfets using dissipative quantum transport simulations. IEEE Electron Device Letters, 33(2):194-196, 2012.

[9] MV Fernández-Serra, Ch Adessi, and X Blase. Surface segregation and backscattering in doped silicon nanowires. Physical review letters, 96(16):166805, 2006.

[10] Hartwin Peelaers, Bart Partoens, and Francois M Peeters. Formation and segregation energies of $\mathrm{b}$ and $\mathrm{p}$ doped and bp codoped silicon nanowires. Nano letters, 6(12):2781-2784, 2006.

[11] Abhishek Kumar Singh, Vijay Kumar, Ryunosuke Note, and Yoshiyuki Kawazoe. Effects of morphology and doping on the electronic and structural properties of hydrogenated silicon nanowires. Nano Letters, 6(5):920-925, 2006.

[12] MT Björk, BJ Ohlsson, Claes Thelander, AI Persson, Knut Deppert, LR Wallenberg, and Lars Samuelson. Nanowire resonant tunneling diodes. Applied Physics Letters, 81(23):4458-4460, 2002.

[13] JJ Gu, YQ Liu, YQ Wu, Robert Colby, Roy Gerald Gordon, and Peide D Ye. First experimental demonstration of gate-all-around iii-v mosfets by top-down approach. In Electron Devices Meeting (IEDM), 2011 IEEE International, pages 33-2. IEEE, 2011.

[14] Fei Xue, Aiting Jiang, Yen-Ting Chen, Yanzhen Wang, Fei Zhou, Yao-Feng Chang, and Jack Lee. Excellent device performance of $3 \mathrm{~d}$ in 0.53 ga 0.47 as gate-wrap-around field-effect-transistors with high-k gate dielectrics. In Electron Devices Meeting (IEDM), 2012 IEEE International, pages 27-5. IEEE, 2012.

[15] Kelin J Kuhn, Martin D Giles, David Becher, Pramod Kolar, Avner Kornfeld, Roza Kotlyar, Sean T Ma, Atul Maheshwari, and Sivakumar Mudanai. Process technology variation. IEEE Transactions on Electron Devices, 58(8):2197-2208, 2011.

[16] Bruce E Kane. A silicon-based nuclear spin quantum computer. nature, 393(6681):133, 1998.

[17] AJ Skinner, ME Davenport, and Bruce E Kane. Hydrogenic spin quantum computing in silicon: a digital approach. Physical review letters, 90(8):087901, 2003.

[18] Daniel Loss and David P DiVincenzo. Quantum computation with quantum dots. Physical Review A, 57(1):120, 1998.

[19] José M Soler, Emilio Artacho, Julian D Gale, Alberto García, Javier Junquera, Pablo Ordejón, and Daniel Sánchez-Portal. The siesta method for ab initio order-n materials simulation. Journal of Physics: Condensed Matter, 14(11):2745, 2002.

[20] John P Perdew, Kieron Burke, and Matthias Ernzerhof. Generalized gradient approximation made simple. Physical review letters, 77(18):3865, 1996.

[21] Xiang-Yang Liu, Wolfgang Windl, Keith M Beardmore, and Michael P Masquelier. First-principles study of phosphorus diffusion in silicon: Interstitial-and vacancy-mediated diffusion mechanisms. Applied physics letters, 82(12):1839-1841, 2003.

[22] Norman Troullier and José Luís Martins. Efficient pseudopotentials for plane-wave calculations. Physical review B, 43(3):1993, 1991.

[23] John P Perdew. Density functional theory and the band gap problem. International Journal of Quantum Chemistry, 28(S19):497-523, 1985.

[24] PS Yadav, RK Yadav, S Agrawal, and BK Agrawal. Ab initio study of silicon in gw approximation: A direct band gap semiconductor. Progress in crystal growth and characterization of materials, 
52(1-2):10-14, 2006.

[25] Mark van Schilfgaarde, Takao Kotani, and Sergey Faleev. Quasiparticle self-consistent g w theory. Physical review letters, 96(22):226402, 2006.

[26] A Svizhenko, MP Anantram, TR Govindan, B Biegel, and R Venugopal. Two-dimensional quantum mechanical modeling of nanotransistors. Journal of Applied Physics, 91(4):2343-2354, 2002.

[27] R Venugopal, Z Ren, S Datta, MS Lundstrom, and D Jovanovic. Simulating quantum transport in nanoscale transistors: Real versus mode-space approaches. Journal of Applied physics, 92(7):3730-3739, 2002.

[28] A. Martinez, A. R. Brown, A. Asenov, and N. Seoane. A comparison between a fully-3d real-space versus coupled mode-space negf in the study of variability in gate-all-around si nanowire mosfet. In 2009 International Conference on Simulation of Semiconductor Processes and Devices, pages 1-4, Sept 2009.

[29] A Martinez, M Bescond, AR Brown, JR Barker, and A Asenov. A full 3d non-equilibrium green functions study of a stray charge in a nanowire mos transistor. Journal of Computational Electronics, 7(3):359-362, 2008.

[30] A. Price and A. Martinez. Investigation on phonon scattering in a GaAs nanowire field effect transistor using the non-equilibrium green function formalism. Journal of Applied Physics, 117(16):164501, 2015. 